\title{
Calibration method for seismic acoustic transducers for rock mass control
}

\author{
Igor Krivosheyev ${ }^{1 *}$, Marina Ignatieva ${ }^{2}$ and Elena Krivosheyeva ${ }^{3}$ \\ ${ }^{1}$ Mining Institute FEB RAS, 51 Turgenev st., Khabarovsk, 680000, Russia; \\ ${ }^{2}$ Far Eastern Department of Rostechnadzor, 680000, Khabarovsk, Russia; \\ ${ }^{3}$ Federal State Budgetary Institution of Education Pacific State University, 680000, Khabarovsk, \\ Russia
}

\begin{abstract}
A new method for calibrating seismoacoustic transducers is proposed, which is free from well-known drawbacks that affect the reliability of measurements. A functional diagram is proposed for calibrating seismoacoustic transducers with improved reliability using a fiber-optic interferometer and high sensitivity due to the possible choice of the type of interferometer.
\end{abstract}

\section{Introduction}

In most cases, when monitoring various structures and controlling the failure of solids under load, such as rock mass, researchers use seismoacoustic transducers (SAT) from different manufacturers or own-made. And it is quite obvious that the manufacturer is obliged to attach appropriate documents to such SAT indicating their characteristics. When using such SAT in information systems [1-4], it is assumed that their characteristics are the same to obtain reliable results. However, the manufacturer does not always provide the data sheet with the measured characteristics of such SAT. Often, the passport contains the average characteristics, or the minimum (maximum) possible. For the successful operation of information-measuring systems, it is often necessary to have a SAT with the same characteristics, for example, to locate sources of destruction in various materials and structures, when measuring the energy indicators of destruction, etc. Existing methods and devices for monitoring SAT characteristics are not devoid of various kinds of errors that significantly affect the measurement of SAT characteristics, and, actually, the reliability of various measurements and controls.

When measuring various characteristics of SAT, directly in the part where surface displacement is monitored, two-beam laser interferometers are used [3-6]. However, in these devices, laser interferometers measure or control the displacement of the surfaces on which the transducers are mounted. In various devices, these surfaces are located next to the SAT or on the back of the rock mass base on which they are installed. When calibrating the SAT, two transducers are used: the reference and the calibrated ones. In this case, it is necessary to measure displacements at two points in the immediate vicinity of the installed

\footnotetext{
*Corresponding authors: igork@as.khb.ru
} 
transducers. To create the same displacements of the working surfaces of the reference and the calibrated transducers, it is necessary to change the place of application of the emitting signal and its power. But in this case, it is not the displacement of the working surfaces [7, 8] of the reference and the calibrated transducers being measured, but the displacement of some point near their location. Moreover, in this case, the added mass is not taken into account (SATs may have a mass different from each other) and, as a consequence, the acoustic contact of the transducer with the rock mass base. All this makes such devices unreliable and poorly usable in practice. Below is a method [9] and a variant of the device [10] for calibrating SAT free of the above disadvantages.

\section{Method and device}

In connection with the above, a method to some extent free from the above comments and disadvantages was developed $[8,9]$. Its essence is as follows. First of all, it is necessary to change the point that the laser interferometer controls. Whatever place one takes on a monolithic block to control mechanical vibrations, nothing can be said about mechanical movements or vibrations of the SAT working surface without proper mathematical processing. This means that it is necessary to control the direct mechanical displacement of the SAT working surface. For this, it is proposed to use a small hole in a monolithic block. Because SATs operate in the frequency range from several hundred hertz to several tens of kilohertz, their dimensions differ significantly from transducers that control acoustic emission. The choice of the diameter of the small hole is proposed to be made from the ratio $D / d>200$, where $D$ is the diameter of the working surface of the SAT used. With this approach, the use of a simple laser interferometer is very difficult, because the difference in the arms of the path of optical beams will not allow using such an interferometer to the fullest extent due to the large error and, ultimately, low accuracy. Here, fiber-optic laser interferometers (FOLI) would be the closest fit. In their case, one can choose the diameter of a small hole in a monolithic slab of about one millimeter, because single-mode optical fiber such as the G.652 standard is much thinner than that. When using FOLI, one can also choose the type of interferometer (Michelson, Fabry-Perot, etc.) with sensitivity suitable for measurements.

According to the proposed method, a device was developed $[9,10]$, the functional block diagram of which is shown in Fig. 1. Here, 1 - optical quantum generator (OQG), 2 optical splitter, 3 - optical fiber, 4 - photodetector, 5 - receiving device, 6 - reference seismoacoustic transducer (SAT), 7 -calibrated SAT, 8 - monolithic block, 9 - mosaic emitter, 10 - voltage regulating elements, 11 - solver, 12 - generator, 13 - electrical signal meter, 14 - recorder, 15 - comparator, 16 - fiber-optic multi-beam interferometer.

The calibrated and the reference SATs 6, 7 are installed on the monolithic block 8 with the centers of their working surfaces on the holes made in 8 , in which fiber-optic multibeam interferometers 16 are installed. The outputs of the multi-beam optical interferometers 16 are connected to the inputs of the comparator 15 through the photodetectors 4 and the receiving devices 5 . The mosaic emitter 9 is rigidly fixed on the reverse side of the monolithic block 8 and is connected to the generator 12 through the voltage regulating elements 10 . The outputs of the reference and the calibrated SATs are connected to the first inputs of the recorders 14, where the generator 12 is connected to the second inputs, and the control inputs of the recorders 14 are connected to the output of the comparator 15 and the input of the solver 11, the outputs of which are connected to the control inputs of the voltage regulating elements 10 , whose main inputs are connected to the generator 12 .

The device works as follows. The generator 12 excites the radiating element 9 fixed on the monolithic block 8, which emits mechanical vibrations. The reference 6 and the 
calibrated 7 SATs receive mechanical displacement and convert it to electrical output. The mechanical displacement of the working surfaces of the reference 6 and the calibrated 7 SATs is directly measured by two multi-beam fiber-optic interferometers 16 .

The use of two-beam interferometers as an interference meter of mechanical displacements [1,2] is ineffective, because a large unbalance in the arms of such interferometers leads to a decrease in sensitivity, and, ultimately, to a significant error and impossibility of taking measurements. In this case, it is advisable to use a multi-beam optical interferometer as an interference meter for mechanical displacements with the ability to measure the SAT vibrational surface through the hole. Multi-beam fiber-optic laser interferometers offered in this solution are best suited for this task.

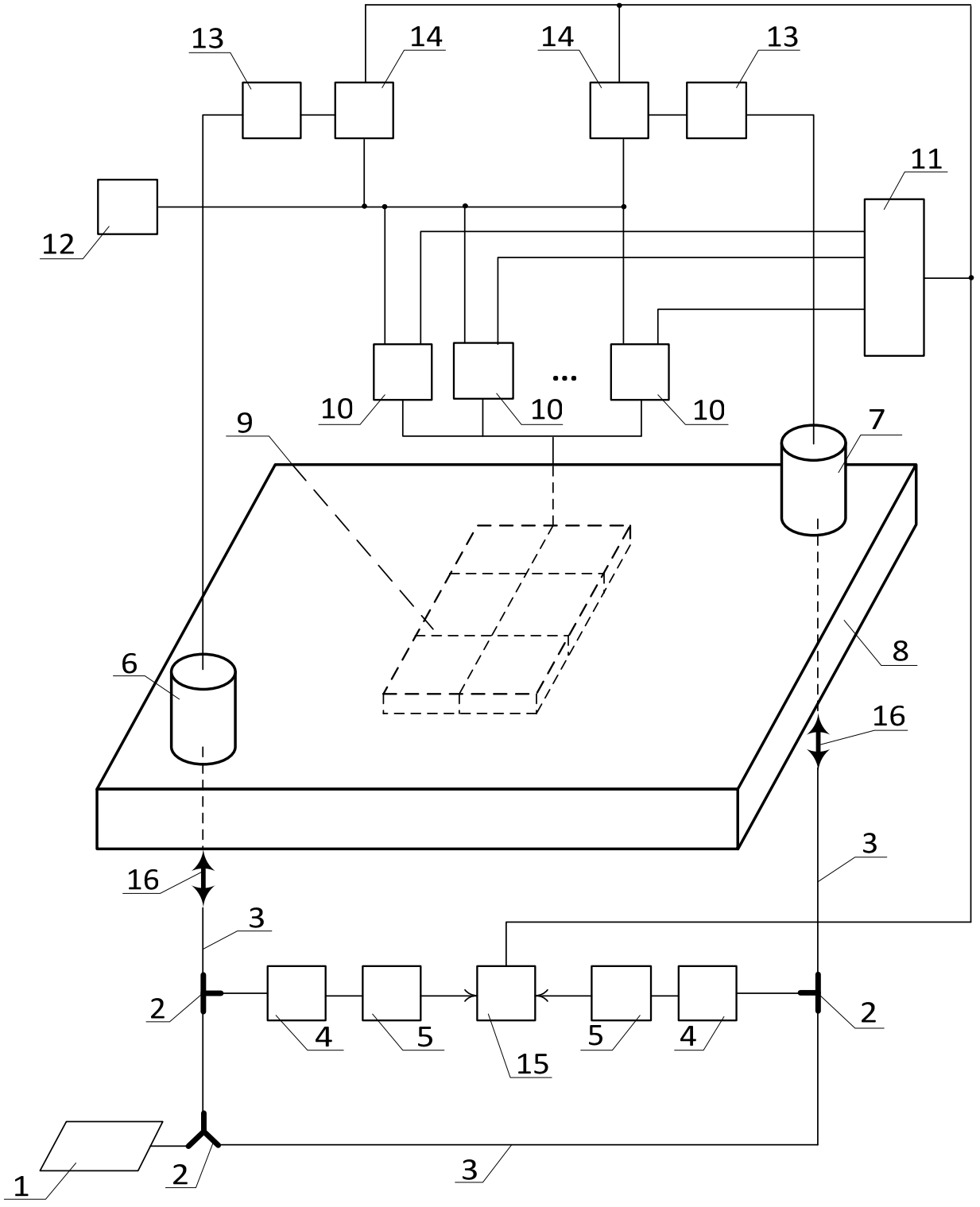

Fig.1.Functional block diagram 
The results of measurements of mechanical displacements of the working surfaces of the reference 6 and calibrated 7 SATs through receiving devices 5 and photodetectors 4 are fed to the compared inputs of the comparator 15, the output of which is connected to the input of the solver 11 . The solver 11 controls the voltage regulating elements 10 on the elements of the mosaic emitter 9 to achieve the same mechanical displacement of the working surfaces of the reference 6 and the calibrated 7 SATs. Upon reaching the same displacement of the working surfaces of 6 and 7 SATs, a voltage is generated at the output of the comparator 15 , which allows the recorders 14 to automatically record electrical signals from the outputs of the reference 6 and the calibrated 7 SATs throughelectrical signals meters 13 , with a fixed value of the generator 12 , and at the same time, without changing the settings of the elements 10 that regulate the radiation voltage. Fiber-optic multi-beam interferometers 16 are mechanically decoupled from the monolithic block 8 .

Thus, the seismoacoustic transducers are calibrated in automatic mode.

\section{Discussion of results and conclusions}

The method itself and the proposed device compare favorably with the known ones by increasing the reliability due to direct control of the working surfaces of the reference and the calibrated SATs using the proposed fiber-optic laser interferometer, and also due to the use of a matrix emitting element, which does not have to be moved over the surface of the monolithic block and control its acoustic contact. The complexity of this approach is justified by obtaining a reliable measurement result. Technically, the device can be simplified by combining some functional blocks, but in principle, this approach is justified.

Separate optical elements have been tested, which made it possible to assess the sensitivity of the proposed method, as well as the acoustic path together, enabling to eliminate the influence of the acoustic contact of both the SAT with the monolithic block and the mosaic structure. The FOLI head itself was made of a multimode optical fiber of the G.652 type with a core diameter of $8 \mu \mathrm{m}$ and was placed in a hole in the monolithic block $1 \mathrm{~mm}$ in diameter.

This method can be proposed for implementation in laboratories designed to measure various characteristics of seismoacoustic transducers, and in metrological services.

\section{References}

1. I.A. Krivosheev, Defectoscopy, 9, 34-38 (2009)

2. I.A. Parinov, A.V. Popov, E.V. Rozhkov et al., Defectoscopy, 1, 66-71 (2000)

3. A.V. Erofeev and V.I. Pasechnik, Acoustic journal, 4(41), 642-643 (1995)

4. Zh. Zhelkobaev, V. Kalendin, P. Todua, Photonics, 5, 14-17 (2009)

5. B.G. Vladimirov, Zh. Zhelkobaev V. Kalendin et al. A method for calibrating acoustic emission transducers and a device for its implementation. RF Patent No. 2321840 (2008)

6. USStandardE076-94

7. I.A. Krivosheev, M.I. Ignatieva, A.I. Shamurina, Informatics and control systems, 2 , 168-172 (2014)

8. I.A. Krivosheev, N.D. Povarov, Informatics and control systems, 3, 112-117 (2019)

9. I.A. Krivosheev, M.I. Ignatieva, Method for calibrating seismoacoustic transducers. RF patent No. 2612272 (2017)

10. I.A. Krivosheev, M.I. Ignatiev, A device for calibrating seismoacoustic transducers. RF Patent No. 2618497 (2017) 